\title{
Editorial
}

\section{Ischaemia-reperfusion: time to increase our awareness during anaesthesia}

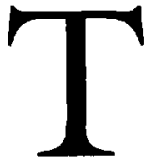

HE pathogenesis of neural injury following ischaemia-reperfusion (IRI) is not completely understood. Two principal mechanisms of injury have been considered: i) oxidant damage from oxygen derived free radicals (ODFRs) and ii) the action of excitatory amines. ${ }^{1}$ The main thrust of research has been to identify injurious species and the prevention of their effects by a variety of approaches including enzymatic degradation or antagonist therapy. The natural history of CNS IRI is poorly defined. It is thought to be initiated in less than one hour, often in seconds to minutes. ${ }^{2}$ This coincides with the release of ODFRs and their effects on lipid peroxidation, i.e., the "respiratory burst."

In this issue of the Journal, Yamaguchi et al. describe delayed neural damage coincident with the detection of the superoxide radical. These changes occurred beyond $72 \mathrm{hr}$, a time usually associated with the extension of injury. If the damaging effects of IRI extend some five to seven days following insult, this finding has clinical implications and expands our understanding of the natural history of this phenomenon. This study suggests the possibility of a process or processes that are not limited or static, but involved in the sustained generation of ODFRs and their damaging effects.

The authors offer two explanations for their findings: activation of the xanthine oxidase system and neutrophils in the area of injury. Although contributory and fitting the time frame described, these mechanisms may not be the complete answer. Other possible causes for the extension of injury that have not been considered include:

1. ODFR activation of neutrophil-platelet interactions resulting in the release of lipid derived inflammatory mediators such as thromboxane or platelet activating factor.
2. Neutrophil-platelet activated release of inflammatory mediators, such as cytokines or nitric oxide, following adhesion with endothelium.

3. Activation of alternate pathways of ODFR generation.

4. Exposure to halogenated anaesthetics.

While the immediate focus of this study was to correlate neural injury with superoxide radical formation, the findings may represent part of a larger series of mechanisms at work that resulted in neural injury over a matter of days.

The "respiratory burst" is hypothesised to initiate the activation of a variety of immune responses over hours to days that would contribute to tissue injury. ${ }^{3}$ Both neutrophils and platelets play key roles in the amplification of this response. ${ }^{4}$ Platelets may be a source of the sustained and continuous generation of ODFRs via the action of platelet activating factor. Neutrophils interact with platelets to enhance this response. The extravasation of red blood cells into areas of tissue disruption would provide a ready supply of iron and copper, catalysts for the sustained enzymatic generation of toxic oxidant species.

Peroxidases may utilise $\mathrm{H}_{2} \mathrm{O}_{2}$ (peroxide) derived from the dismutation of the superoxide radical following the respiratory burst to produce substances many times more toxic than either superoxide or peroxide. ${ }^{5}$ Myeloperoxidase (MPO), supplied from activated neutrophils and monocytes, catalyses the oxidation of halides $\left(\mathrm{Cl}^{-}, \mathrm{Br}^{-}, \mathrm{F}^{-}\right)$to produce hypohalous acids. Hypohalous acid is a very potent oxidant. The hypohalous acid derived, can combine with biological amines, increasing their oxidant capacity, as the final mediators of MPO dependent cell damage.

Address correspondence to: Dr. David M. Ansley, Department of Anaesthesia, Room 3200, 3rd Floor, 910 West 10th Avenue, Vancouver, BC, Canada V5Z 4E3. 
This consideration is important because of the possibility that halogenated anaesthetics may support such processes. Oxidant mediated injury has been shown to be enhanced by halothane and isoflurane. ${ }^{6}$ The effects with halothane may be the most potent and may be dose-dependent. These anaesthetics serve as substrate for the production of their own free radicals. ${ }^{7}$ They may act as a source of $\mathrm{Cl}^{-}, \mathrm{Br}^{-}$, and $\mathrm{F}^{-}$ moieties involved in electron transfers responsible for the alternate generation of ODFRs or production of hypohalous acids that could extend the injury process. Alternatively, these anaesthetic vapours may increase the susceptibility of endothelial cells to oxidant damage. The exact mechanism has not been identified but may occur via antioxidant enzymatic inhibition, or disturbances in $\mathrm{Ca}^{++}$and $p H$ homeostasis at the level of the cell membrane. ${ }^{6}$

It is likely these effects do not act as isolated reactions but combine in intricate biofeedback loops that ensure the ongoing generation of a variety of ODFRs that contribute to the delayed or extended injury described by Yamaguchi et al. The CNS is particularly prone to these effects given its lipid and mitochondrial rich environment, which would act as both the supply and source of production of many of the damaging substances described. Oxygen is very soluble in the brain and the framework to supply the cellular components of ODFR generation is readily available via a rich supply of delicate blood vessels.

The importance of the work of Yamaguchi $e t a l$. is in identifying a delayed process of injury though secondary to the superoxide radical. Our understanding of the pathogenesis and natural history of CNS IRI is enhanced by such findings. Like any good investigation, it creates more questions than answers. Clinically, their findings suggest new considerations in the prevention and management of free radical tissue injury. Extended treatment over several days may be warranted. Alternatively, a multifactorial approach that could limit the amplification of the injury response may be indicated. Research in these areas continues.

In addition, this study begs the question of what, if any, contribution anaesthetic choice may have in the pathogenesis of IRI. If there were a way to exclude volatile anaesthetics from the methodology, would their results have been different? Drugs like isoflurane are generally considered neuroprotective. The potential to act as catalyst for the generation of ODFRs and other mediators of injury has received only recent attention. This potential paradox deserves further consideration in the design and conduct of both laboratory and clinical investigation.

\section{L'ischémie-reperfusion : c'est le temps d'être plus conscient de ce problème durant d'anesthésie}

La pathogénèse du dommage neurologique associé à l'ischémic-reperfusion (IRI) n'est pas complètement comprise. Deux mécanismes principaux ont été envisagés: 1) des dommages oxydatifs dus aux radicaux libres dérivés de l'oxygène (ODFRs) et 2) l'effet des amines excitatives'. L'objectif principal de la recherche a été d'identifier les variétés dommageables et la prévention de leurs effets par une variété d'approches incluant la dégradation enzymatique et la thérapie antagoniste. L'évolution naturelle des IRI du SNC est mal définie. On croit que l'IRI s'initie en moins d'une heure, souvent sur une période de secondes ou de minutes ${ }^{2}$. Ceci coïncide avec la relâche des ODFRs ainsi qu'avec leurs effets sur la peroxydation lipidique, i.e., la «bouffée oxydative».

Dans ce numéro du Journal, Yamaguchi et al. décrivent des dommages neuraux retardés coïncidant avec la détection de radical superoxyde. Ces dommages sont survenus après 72 heures, alors que cette période est normalement associée avec la fin de l'extension des lésions. Si les effets dommageables de l'IRI progressent durant 5 à 7 jours à la suite de l'insulte, cette découverte a des implications cliniques et accroit notre compréhension de l'histoire naturelle de ce phénomène. Cette étude suggère la possibilité d'un ou de procédés qui ne sont ni limités ni statiques mais qui sont impliqués dans la production continue des ODFRs et de leurs effets dommageables.

Les auteurs présentent deux explications à leurs trouvailles: activation du système xanthine oxydase ainsi qui des neutrophiles dans la zone lésée. Même s'ils sont impliqués et qu'ils respectent l'intervalle de temps décrit, ces mécanismes peuvent ne pas représenter la réponse complète. Les autres causes possibles d'extension de la lésion qui n'ont pas été envisagées sont:

1. L'activation par les ODFRs des interactions neutrophiles-plaquettes résultant dans la relâche des médiateurs de l'inflammation dérivés des lipides comme la thromboxane ou le facteur d'activation des plaquettes.

2. La relâche de médiateurs inflammatoires activée par les neutrophiles-plaquettes tels les cytoxines 
ou l'oxyde nitrique, suite à leur adhésion à l'endothélium.

3. L'activation des voies alternatives de génération des ODFRs.

4. L'exposition aux agents anesthésiques halogénés.

Bien que le but premier de cette étude ait été de relier la lésion neurale à la formation de radicaux superoxyde, les résultats peuvent représenter une partie d'une série plus vaste de mécanismes responsables de lésions neurales progressant sur plusieurs jours.

La «bouffée oxydative» est proposée comme mécanisme initiant l'activation d'une variété de réponses immunes s'étendant de plusieurs heures à plusieurs jours et contribuant au dommage des tissus ${ }^{3}$. Les neutrophiles autant que les plaquettes jouent un rôle clé dans l'augmentation de cette réponse ${ }^{4}$. Les plaquettes peuvent être une source de génération soutenue et continue de ODFRs par l'action du facteur activant les plaquettes. Les neutrophiles réagissent avec les plaquettes pour augmenter cette réponse. L'extravasation des globules rouges dans les zones de destruction tissulaire fournirait un apport de fer et de cuivre, catalyseurs nécessaires à une génération enzymatique soutenue d'une variété d'oxydants toxiques.

Les peroxydases peuvent utiliser l' $\mathrm{H}_{2} \mathrm{O}_{2}$ (peroxyde) provenant de la dismutation du radical superoxyde suite à la «bouffée oxydative» pour produire des substances plusieurs fois plus toxiques que le superoxyde ou le peroxyde ${ }^{5}$. La myéloperoxydase (MPO) fournie par les neutrophiles activés et les monocytes, catalyse l'oxydation des halogènes $\left(\mathrm{Cl}^{-}, \mathrm{Br}^{-}, \mathrm{F}^{-}\right)$pour produire les acides halogénés correspondants. Les acides halogénés sont des oxydants très puissants qui peuvent se combiner avec les amines biologiques, augmentant leur capacité oxydative comme médiateurs finaux du dommage cellulaire provoqué par la MPO.

Cette considération est importante parce qu'il est possible que les anesthésiques halogénés supportent de tels processus. On a montré que les dommages médiés par les oxydants étaient aggravés par l'halothane et l'isoflurane ${ }^{6}$. Les effets dus à l'halothane sont peut-être les plus puissants et sont peut-être dépendants de la dose. Ces anesthésiques servent de substrats pour la production de leurs propres radicaux libres ${ }^{7}$. Ils peuvent agir comme source des parties $\mathrm{Cl}^{-}, \mathrm{Br}^{-}, \mathrm{F}^{-}$impliquées dans les transferts d'ćlectrons responsables d'une voie alternative de génération des ODFRs ou de production des acides halogénés qui peuvent augmenter le processus lésionnel. Ces anesthésiques pourraient aussi augmenter la susceptibilité des cellules endothéliales aux dommages oxydatifs. Le mécanisme exact n'a pas été identifié mais il pourrait s'agir d'une inhibition enzymatique antioxydante ou de perturbations de l'homéostase du $\mathrm{Ca}^{++}$et du $p H$ au niveau de la membrane cellulaire.

Il est probable que ces effets ne surviennent pas comme des réactions isolées mais qu'ils se combinent en des boucles rétroactives compliquées, assurant ainsi la génération d'une variété de ODFRs qui contribuent aux lésions étendues ou retardées décrites par Yamaguchi et al. Le SNC est particulièrement susceptible à ces effets compte tenu de son environnement lipidique riche en mitochondries, cet environnement agissant comme fournisseur et producteur de plusieurs des substances dommageables déjà décrites. L'oxygène est très soluble dans le cerveau, et la trame nécessaire aux composantes cellulaires produisant l'ODFR se retrouve dans le riche réseau de fins vaisseaux sanguins.

L'importance du travail de Yamaguchi et al. réside dans l'identification d'un processus de lésion retardé, en relation avec le radical superoxyde. Notre compréhension de la pathogénèse et de l'histoire naturelle des IRI du SNC est améliorée par leurs trouvailles. Comme toute bonne recherche, elle suscite plus de questions que de réponses. Au point de vue clinique, leurs trouvailles suggèrent de nouvelles considérations dans la prévention et le traitement des lésions tissulaires par les radicaux libres. Un traitement prolongé sur plusieurs jours pourrait être indiqué. D'autre part, on pourrait privilégier une approche multifactorielle de façon à limiter l'amplification de la réponse à l'insulte originale. Les recherches sur ce sujet continuent.

En outre, cette étude pose la question à savoir quelle est la contribution du choix des agents anesthésiques à la pathogénèse de l'IRI. Si leur méthodologie avait pu exclure les agents anesthésiques, est-ce que leurs résultats auraient été différents? Des médicaments comme l'isoflurane sont en général considérés comme neuroprotecteurs. Leur potentiel d'agir comme des catalyseurs dans la production de ODFRs et d'autres médiateurs de lésions tissulaires n'a reçu d'attention que récemment. Ce paradoxe potentiel doit être pris en considération dans la mise au point et la conduite des études tant en laboratoire qu'en clinique.

\section{References}

1 Hurn PD, Kirsch JR, Traystman RJ. Pharmacologic neuroprotection: fact or fantasy? In: Blanck TJJ (Ed.). Neuroprotection. Baltimore: Williams \& Wilkins, 1997: 47-62.

2 Fehér J, Csomós G, Vereckei A. Physiological free radical reactions. Free Radical Reactions in Medicine. Berlin: Springer-Verlag, 1987: 18-39. 
3 Herskowitz $A$, Mangano $D T$. Inflammatory cascade - a final common pathway for perioperative injury? (Editorial) Anesthesiology 1996; 85: 957-60.

4 Faint $R W$. Platelet-neutrophil interactions: their significance. Blood Rev 1992; 6: 83-91.

5 Grisham $M B, M c$ Cord J. Chemistry and cytotoxicity of reactive oxygen metabolites. In: Taylor $\mathrm{AE}$, Matalon $S$, Ward PA (Eds.). Physiology of Oxygen Radicals, American Physiological Society. Baltimore: Williams \& Wilkins, 1986: 1-19.

6 Shayevitz JR, Varani J, Ward PA, Knight PR. Halothane and isoflurane increase pulmonary artery endothelial cell sensitivity to oxidant-mediated injury. Anesthesiology 1991; 74: 1067-77.

7 Lal M, Schoneich C, Monig J, Asmus K-D. Rate constants for the reactions of halogentaed organic radicals. Int J Radiat Biol 1988; 54: 773-85. 